\title{
Magnetophoresis of Tagged Polymers
}

\author{
G. T. Barkema \\ Institute for Advanced Study, Olden Lane, Princeton NJ 08540, USA \\ G. M. Schütz \\ Theoretical Physics, University of Oxford, \\ 1 Keble Road, Oxford, OX1 3NP, United Kingdom.
}

\begin{abstract}
We present quantitative results for the drift velocity of a polymer in a gel if a force (e.g. through an electric or magnetic field) acts on a tag, attached to one of its ends. This is done by introducing a modification of the RubinsteinDuke model for electrophoresis of DNA. We analyze this modified model with exact and Monte Carlo calculations. Tagged magnetophoresis does not show band collapse, a phenomenon that limits the applicability of traditional electrophoresis to short polymers.
\end{abstract}

Typeset using REVTEX 
If electrophoresis is applied for the separation of large polymers, for instance fragments of DNA, the velocity becomes independent of the length. This phenomenon is called "band collapse" and is widely known amongst experimentalists. A consequence is that long polymers cannot be separated efficiently as they will all travel with approximately the same velocity. Surprisingly, these experimental results can be predicted from a quite simple onedimensional lattice gas model, proposed by Rubinstein [1] as a model for the dynamics of entangled polymers (the repton model), and adapted by Duke as a model for gel electrophoresis of DNA [2]. It has been studied both analytically [1,3, 团, and by Monte Carlo simulations [2:35.5]. The same lattice gas model, but seen in a different context, is of interest in other areas: it describes coarsening [6], diffusion of hard-core particles [7] with open boundary conditions, and interface growth [8].

In this work we focus on its application to the motion of long polymers in a gel. We study a modification of the traditional electrophoresis: instead of pulling uniformly charged polymers with an electric field, we propose to pull on one of the ends of the polymer, which can be realized by attaching a magnetic tag to the polymer and applying a magnetic field. We will show that according to the repton model, in this case the drift velocity stays lengthdependent, allowing for the efficient separation of long polymers. In previous work on the application of tags in electrophoresis [9], a force acts on the polymer and not on the tag, whereas we propose to pull on the tag and not on the polymer.

First we will give a short description of the repton model, list some of its properties, and compare the properties of the repton model with experimental results on DNA electrophoresis in agarose gels. Next, we will use the repton model to study what happens if a force pulls only on one end of the polymer rather than uniformly along the whole molecule. Finally, we will outline some possible experimental realizations and summarize our results.

The repton model is illustrated in Fig. 1. In this model, the gel is represented as a collection of pores that are arranged in a square lattice. A polymer, represented as a chain of $N$ polymer segments or reptons (the black dots in Fig. 1) moves through this gel.

These reptons move from pore to pore diagonally according to the following rules: 
(a) reptons in the interior of the chain move only along the sequence of pores occupied by the chain. This restriction ensures that the only possible mechanism of movement is reptation. (b) at least one repton must remain in each pore along the chain but otherwise the number of reptons in a pore is unrestricted. By imposing this rule we achieve that the polymer has some elasticity but does not stretch to infinite length.

(c) the two chain end segments may move to adjacent pores provided that the rule (b) is not violated.

The dynamics of the model are defined as follows: Each possible move (each repton moving in any direction) is tried with a constant rate, setting the unit of time. This represents the diffusive motion of the polymer. To make it also a model for electrophoresis, Duke has introduced a small modification to this original repton model. To each repton a negative electric charge is assigned on which the electric field $E$ acts, breaking the symmetry. Instead of unit rates for the allowed moves in the positive and negative $x$-direction, the rate for allowed moves in the positive $x$-direction (direction opposite to the electric field) equals $\exp (+E / 2)$, while the rate for allowed moves in the negative $x$-direction equals $\exp (-E / 2)$.

For the application to electrophoresis the quantity of interest is the velocity of the polymer as a function of the field strength and the length of the polymer. In extensive Monte Carlo simulations [5] it was found that a data collapse could be obtained if the rescaled velocity $v N^{2}$ is plotted as a function of the rescaled electric field $N E$. Plotted in such a way, all data points were found to be located on one single curve, given by

$$
\frac{v N^{2}}{\beta}=\left[\left(\frac{N E}{\alpha}\right)^{2}+\left(\frac{N E}{\alpha}\right)^{4}\right]^{1 / 2},
$$

where $\beta=5 / 6$ and, as expected from the Nernst-Einstein relation, $\alpha / \beta=D N^{2}=1 / 3$ where $D$ is the diffusion coefficient of the polymers. For small $N E$, the rescaled velocity $v N^{2}$ increases linearly with $N E$. corresponding to $v=E / 3 N$. For large $N E$, the rescaled velocity shows a quadratic behavior. This corresponds to $v \sim E^{2}$.

It turns out that a collapse of experimental data for DNA electrophoresis in agarose can be achieved with Eq. 1, as illustrated in Fig. 2 for data points published by Heller et al. [10] 
and rearranged as in [11]. Taking the velocity $v$ in $\mathrm{cm} / \mathrm{h}$, the electric field $E$ in $\mathrm{V} / \mathrm{cm}$, and DNA lengths $N$ in kilobase-pair (kb), one gets $\alpha / \beta=(0.5 \pm 0.1) p^{3 / 2}$ and $\beta=(17 \pm 5) p^{-5 / 2}$, where $p$ is the gel concentration in percent agarose by weight.

This surprisingly good agreement of experimental data with the theoretical prediction (1) indicates that the repton model contains all important ingredients for DNA electrophoresis: entanglement of the polymer in the gel, entropic elasticity of the polymer, and reptation as the dominant mechanism of movement.

In this work we use the repton model to study the behavior of polymers in a gel if a force is applied only to one of the ends of the polymers. To do this within the framework of the repton model, we preserve the rules that specify which moves are allowed and which ones are not. We assume that the force acts only on repton 1 . The attempt frequencies are now not uniform along the chain: as in the repton model for DNA electrophoresis as adapted by Duke, repton 1 moves in the positive and negative $x$ direction with rates $q=\exp (E / 2)$ and $q^{-1}=\exp (-E / 2)$, respectively; as in the original repton model of Rubinstein, all allowed moves of all other reptons occur with unit rate.

These dynamical rules may be conveniently rephrased in lattice gas language by writing a master equation for a one-dimensional exclusion process, where the links of the repton chain are mapped to the sites of a one-dimensional lattice with $L=N-1$ sites. For each link $i=1 \ldots L$ (connection between reptons $i$ and $i+1$ ) we distinguish three cases: the link has a negative, a zero, or a positive component in the $x$-direction. We identify these three cases with the presence of an $A$-particle, a vacancy, and a $B$-particle, respectively, on site $i$ of the lattice. By this mapping the information on the motion of the polymer perpendicular to the field gets lost, but this motion is purely diffusive and of no interest for the study of the drift velocity of the polymer in direction of the field. The drift velocity is in particle language given by the difference between the current of $A$-particles and the current of $B$-particles along the chain.

In terms of particles, the dynamics may be described as follows: If one site of a pair of nearest-neighbor sites is occupied by a particle while the other site is empty, this particle 
hops to the empty site with rate 1 . In addition to these hopping events, particles can be created and annihilated at the ends. At site $L, A$ - and $B$-particles are created and annihilated with rate 1 , while at site $1 A$-particles are created and annihilated with rates $q$ and $q^{-1}$ and $B$-particles with rates $q^{-1}$ and $q$, respectively.

By writing this process in a quantum Hamilton formalism, the stochastic time evolution of the system is given by the Hamiltonian $H=b_{1}(q)+b_{L}(1)+\sum_{i=1}^{L-1} u_{i}(1)$ of a spin-1 quantum chain where $b_{i}(q)=q\left(1-n_{i}^{A}-a_{i}^{+}-b_{i}\right)+q^{-1}\left(1-n_{i}^{B}-a_{i}-b_{i}^{+}\right)$, and $u_{i}(q)=q\left(n_{i}^{A} n_{i+1}^{0}+\right.$ $\left.n_{i}^{0} n_{i+1}^{B}-a_{i} a_{i+1}^{+}-b_{i}^{+} b_{i+1}\right)+q^{-1}\left(n_{i}^{0} n_{i+1}^{A}+n_{i}^{B} n_{i+1}^{0}-a_{i}^{+} a_{i+1}-b_{i} b_{i+1}^{+}\right)$. Here $n_{i}^{A} \equiv E_{i}^{11}, n_{i}^{B} \equiv E_{i}^{33}$ and $n_{i}^{0}=1-n_{i}^{A}-n_{i}^{B} \equiv E_{i}^{22}$ are projection operators on states with spin $S^{z}=1,0,-1$ resp. on site $i$, identified with an $A$-particle, vacancy and $B$-particle resp. The operators $a_{i} \equiv E_{i}^{21}, a_{i}^{+} \equiv E_{i}^{12}, b_{i} \equiv E_{i}^{23}$, and $b_{i}^{+} \equiv E_{i}^{32}$ are annihilation and creation operators for $A$ and $B$ particles. $E_{i}^{j k}$ is the $3 \times 3$ matrix with matrix elements $\left(E_{i}^{j k}\right)_{\alpha, \beta}=\delta_{j, \alpha} \delta_{k, \beta}$ acting on site $i$.

The average densities $\left\langle n_{i}^{A, B}\right\rangle$ at time $t$ satisfy the continuity equations $\frac{d}{d t}\left\langle n_{i}^{A, B}\right\rangle=$ $-\left\langle n_{i}^{A, B} H\right\rangle=\left\langle j_{i-1}^{A, B}\right\rangle-\left\langle j_{i}^{A, B}\right\rangle$ with the particle currents

$$
\begin{aligned}
\left\langle j_{i}^{A, B}\right\rangle & =\left\langle n_{i}^{A, B} n_{i+1}^{0}\right\rangle-\left\langle n_{i}^{0} n_{i+1}^{A, B}\right\rangle \quad 1 \leq i \leq L-1 \\
\left\langle j_{L}^{A, B}\right\rangle & =\left\langle n_{L}^{A, B}\right\rangle-\left\langle n_{L}^{0}\right\rangle \\
\left\langle j_{0}^{A}\right\rangle & =q\left\langle n_{1}^{0}\right\rangle-q^{-1}\left\langle n_{1}^{A}\right\rangle \\
\left\langle j_{0}^{B}\right\rangle & =q^{-1}\left\langle n_{1}^{0}\right\rangle-q\left\langle n_{1}^{B}\right\rangle
\end{aligned}
$$

Among the quantities of interest are particle densities and correlations in the steady state of the system, in particular the (space-independent) stationary particle currents $\left\langle j_{i}^{A, B}\right\rangle \equiv$ $j^{A, B}$ which give information on the internal structure of the polymer and which give its average drift velocity $v=j^{A}-j^{B}$. Defining $\mathcal{J}=j^{A}+j^{B}$ and $m_{i}=n_{i}^{A}-n_{i}^{B}$, stationarity of the probability distribution leads to the following relations:

$$
\begin{aligned}
\mathcal{J} & =\left\langle n_{i+1}^{0}\right\rangle-\left\langle n_{i}^{0}\right\rangle \quad(1 \leq i \leq L-1) \\
& =\frac{q+q^{-1}}{2}\left(3\left\langle n_{1}^{0}\right\rangle-1\right)+\frac{q-q^{-1}}{2}\left\langle m_{1}\right\rangle
\end{aligned}
$$




$$
\begin{aligned}
& =1-3\left\langle n_{L}^{0}\right\rangle \\
v & =\left\langle m_{i} n_{i+1}^{0}\right\rangle-\left\langle n_{i}^{0} m_{i+1}\right\rangle \quad(1 \leq i \leq L-1) \\
& =\frac{q-q^{-1}}{2}\left(1+\left\langle n_{1}^{0}\right\rangle\right)-\frac{q+q^{-1}}{2}\left\langle m_{1}\right\rangle \\
& =\left\langle m_{L}\right\rangle
\end{aligned}
$$

(2) and (4) give the exact average density of vacant sites at site $i$ in terms of $\mathcal{J}$

$$
\left\langle n_{i}^{0}\right\rangle=\frac{1}{3}[1-(3 L+1) \mathcal{J}]+\mathcal{J} i
$$

and therefore the probability $1-\left\langle n_{i}^{0}\right\rangle$ of finding only one repton in the pore occupied by the $i^{\text {th }}$ repton. $\mathcal{L}=L-\sum_{i=1}^{L}\left\langle n_{i}^{0}\right\rangle=2 L / 3+\mathcal{J} L(3 L-1) / 6$ gives the average length of the polymer in terms of $\mathcal{J}$. Eqs. (3), (6) and (8) (for $i=1$ ) give 3 linear equations for the four quantities $v, \mathcal{J},\left\langle m_{1}\right\rangle,\left\langle n_{1}^{0}\right\rangle$ relating the currents to the average densities at site 1 . This may be used to derive an exact expression for $v$ in terms of any of the three other expectation values.

Now we study $v$ as a function of $q$ and $N=L+1$. The diffusion coefficient $D$ of the repton chains has not changed from the original model, and is to lowest order in $1 / N$ given by $D=1 /\left(3 N^{2}\right)[[2]$. The force on the chain is now independent of its length and proportional to $E$. Combining this with the Nernst-Einstein relation $v \approx F \cdot D$ yields $v=E / 3 N^{2}$ for small forces that do not disturb the equilibrium configuration of the chain.

For intermediate fields and short chains, we can obtain the drift velocity numerically exactly by computing the eigenvector with eigenvalue 0 of the quantum Hamiltonian $H$. Results for chains with up to 7 reptons $(L=6)$ are presented in Fig. 3. For intermediate fields and longer chains, we study the model by Monte Carlo simulations. We performed these simulations by means of a fast "multi-spin" algorithm as described in an earlier paper [5]. Results are included in Fig. 3.

In the limit of an infinitely strong force, we can solve the model exactly for any $L$. For an infinite creation rate of $A$-particles and correspondingly infinite annihilation rate of $B$ particles on site 1 one has $\left\langle n_{1}^{A}\right\rangle=1$ and $\left\langle n_{1}^{B}\right\rangle=\left\langle n_{1}^{0}\right\rangle=0$. In this limit the stochastic process 
becomes equivalent to a process on a chain on $L^{\prime}=L-1$ sites where $A$ particles are created (annihilated) with rate $1(0)$ on site 2 and where no $B$-particles are created or annihilated. Going through the same calculations as above gives expressions similar to (2-7), but with (3) and (6) replaced by $\mathcal{J}=v=\left\langle n_{2}^{0}\right\rangle$ and the range of definition in (2) and (5) replaced by $2 \leq i \leq L-1$. Together with (\$) (for $i=2$ ) this shows that the velocity approaches a constant that depends on the length of the chain: $v=1 /(3 L-2)=1 /(3 N-5)$. This asymptotic regime starts around $E=3$ as can be seen from the exact and Monte Carlo results shown in Fig. 3 .

Thus, according to this modified repton model, there is no band collapse if we pull on one end of the chain, even in the limit of an infinitely strong field. In this limit, the polymer is not fully stretched but has an average length $\mathcal{L}=L\left(5+(3 L-2)^{-1}\right) / 6 \approx 5 L / 6$.

Experimentally, a realization of pulling only at one end of each polymer, is to attach to the end a magnetic bead. Magnetic beads are produced commercially; for a discussion of their properties and traditional application in biotechnology we refer to [13]. These commercially available magnetic beads are rather large for use in a normal agarose gel, and have the risk to get stuck, but a dense polymer solution may be a suitable replacement for the agarose gel. It is interesting to point out that according to our model, variations in the force on the end of the polymer, (e.g. due to differences in the magnetic beads) do not lead to a significant band widening if the force is sufficiently large, as in that case the drift velocity is independent of the force (see figure 3).

If such "magnetophoresis" turns out to be possible, this experimental approach might also avoid band collapse in RNA electrophoresis and make sequencing of longer fragments possible. Another interesting topic is the behavior of a polymer that experiences a magnetic force on one end, in addition to a uniform electric force on the whole chain.

Considerable effort has been invested into solving the Rubinstein-Duke model for electrophoresis, but to the best of our knowledge no rigorous results have been obtained, except for very short polymers [3] and for a related model with periodic boundary conditions [4]. In zero field the model is equal to the symmetric exclusion process with tagged particles, 
which is (at least) partially integrable by the matrix Bethe ansatz discussed in [14]. An open question is whether open boundary conditions as proposed here leave the system completely integrable; this would allow for a full exact solution of both the model proposed here and the original Rubinstein model.

To conclude: previous work has shown that the Rubinstein-Duke model for DNA electrophoresis provides a good description of experimental results of electrophoresis of DNA fragments in agarose gels [11]. We propose a modification in the model to describe pulling only on the end of the polymer instead of uniformly along the chain. With Monte Carlo simulations and exact calculations we predict that this provides a way of avoiding band collapse, a phenomenon that severely limits the separation of long polymers by traditional electrophoresis. Experimental realizations of this modified model and various applications are discussed.

We thank J.-L. Viovy, C. Heller and S. Margel for interesting comments on experimental realizations. G.M.S. would like to thank C. Godreche for stimulating discussions. This work was supported by the EPSRC under Grant No. GR/J78044 (G.T.B.), and under the Human Capital and Mobility program of the European Community (G.M.S.). 


\section{REFERENCES}

[1] M. Rubinstein, Phys. Rev. Lett. 59, 1946 (1987).

[2] T.A.J. Duke, Phys. Rev. Lett. 62, 2877 (1989); T.A.J. Duke, J. Chem. Phys. 93, 9049 (1990); T.A.J. Duke, J. Chem. Phys. 93, 9055 (1990).

[3] B.Widom, J.L.Viovy and A.D.Défontaines, J.Phys. I France 1, 1759 (1991).

[4] J.M.J. van Leeuwen, J. Phys. I France 1, 1675 (1991); J.M.J. van Leeuwen and A. Kooiman, Physica A 184, 79 (1992).

[5] G.T. Barkema, J.F. Marko and B. Widom, Phys. Rev. E 49, 5303 (1994).

[6] J. Kertész and R. Ramaswamy, Europhys. Lett. 28, 617 (1994).

[7] T. Ligget, Interacting Particle Systems, New York, Springer (1985).

[8] J. Krug and H. Spohn, in: Solids far from Equilibrium, ed. C. Godreche, Cambridge, Cambridge University Press (1991).

[9] L. Ulanovsky, G. Drouin and W. Gilbert, Nature 343, 190 (1990); J. Noolandi, Electrophoresis 13, 394 (1992); P. Mayer, G.W. Slater and G. Drouin, Anal. Chem. 66, 1777 (1994); A. Adjari et al., J. Phys. II France 5, 491 (1995).

[10] C. Heller, T.A.J. Duke and J.L. Viovy, Biopolymers 34, 249 (1994).

[11] G.T. Barkema, C. Caron, and J.F. Marko, to appear in Biopolymers.

[12] M. Prähofer, University of Munich thesis (1994).

[13] B.-I. Haukanes and C. Kvam, Biotechnology 11, 60 (1993).

[14] R.B. Stinchcombe and G.M. Schütz, Europhys. Lett. 29, 663 (1995); R.B. Stinchcombe and G.M. Schütz, Phys. Rev. Lett. 75, 140 (1995). 


\section{FIGURES}

FIG. 1. Left: Two-dimensional representation of the repton model of a long polymer. The reptons (black circles) are located in the pores of the gel (squares of the lattice). Right: projected repton model where the reptons are numbered along the chain, and the $x$-coordinate of repton $i$ is plotted as a function of $i$. The arrows indicate the allowed moves.

FIG. 2. Experimental data on the rescaled drift velocity $v N^{2} / \beta$ as a function of the rescaled electric field $N E / \alpha$. The solid curve is the theoretical prediction by the repton model, see eq. 11. The data points are obtained from [10] and rearranged as in [11].

FIG. 3. Drift velocity $v$ as a function of the force $E$. The solid lines are exact results for

$L=2 \ldots 6$, the dashed lines are obtained from Monte Carlo simulations for $L=7,10,15,20,30$ and 50. Statistical errors smaller than $2 \%$ are omitted. 


\section{Barkema/Schutz Fig. 1}
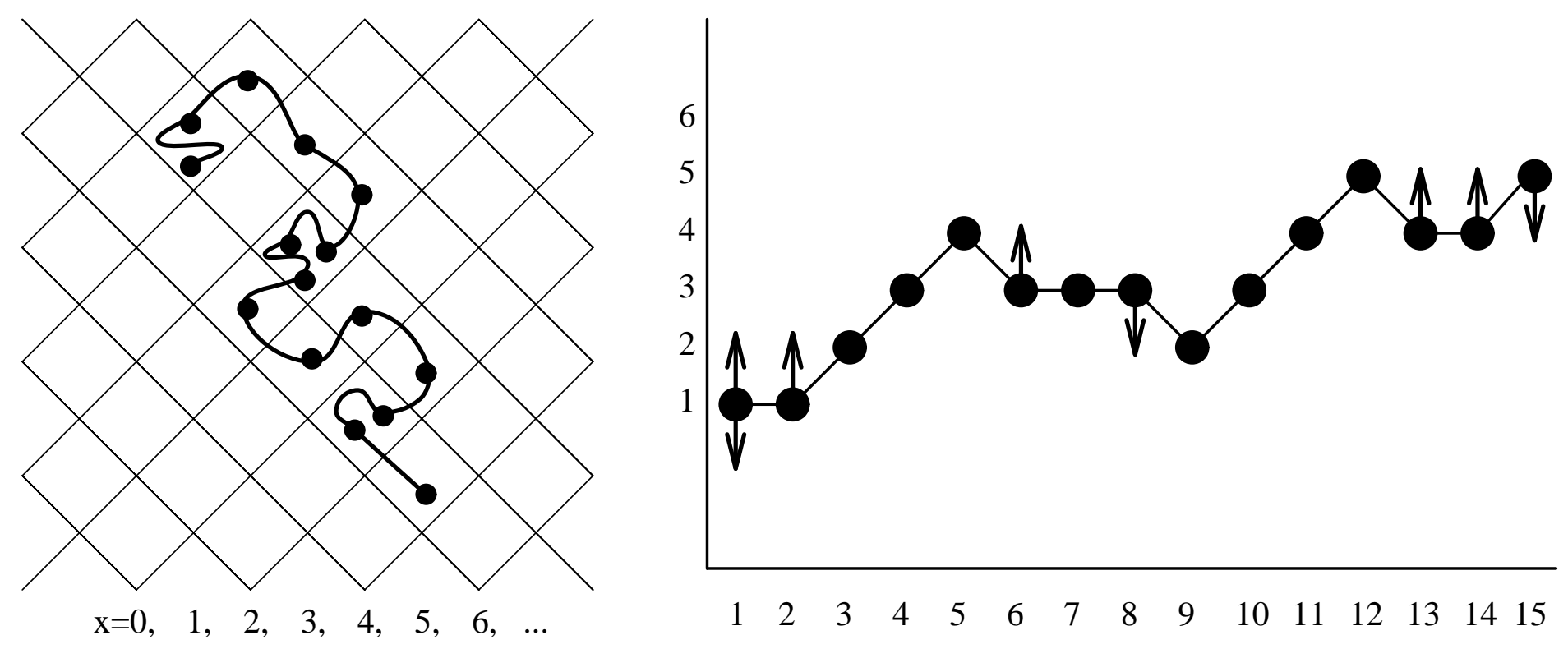


\section{Barkema/Schutz Fig. 2}

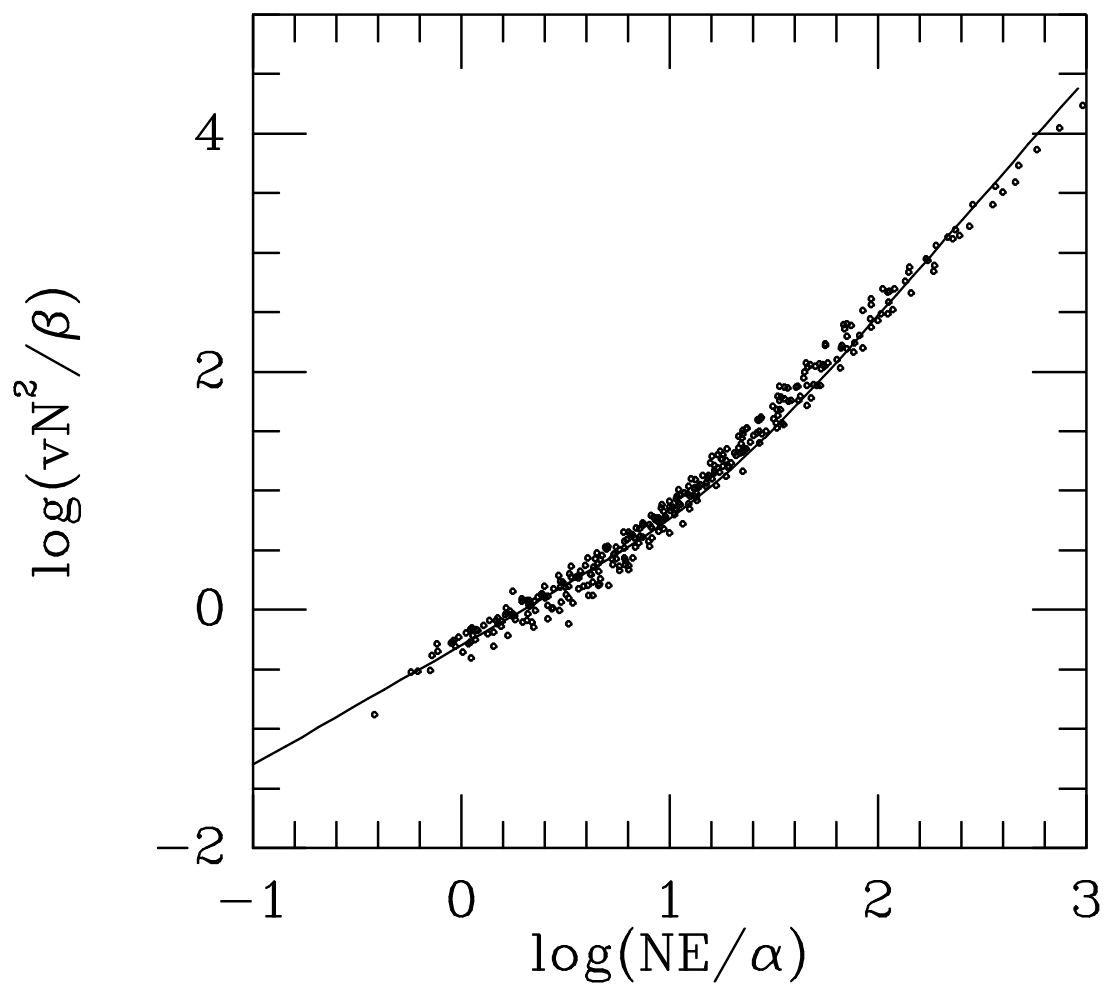




\section{Barkema/Schutz Fig. 3}

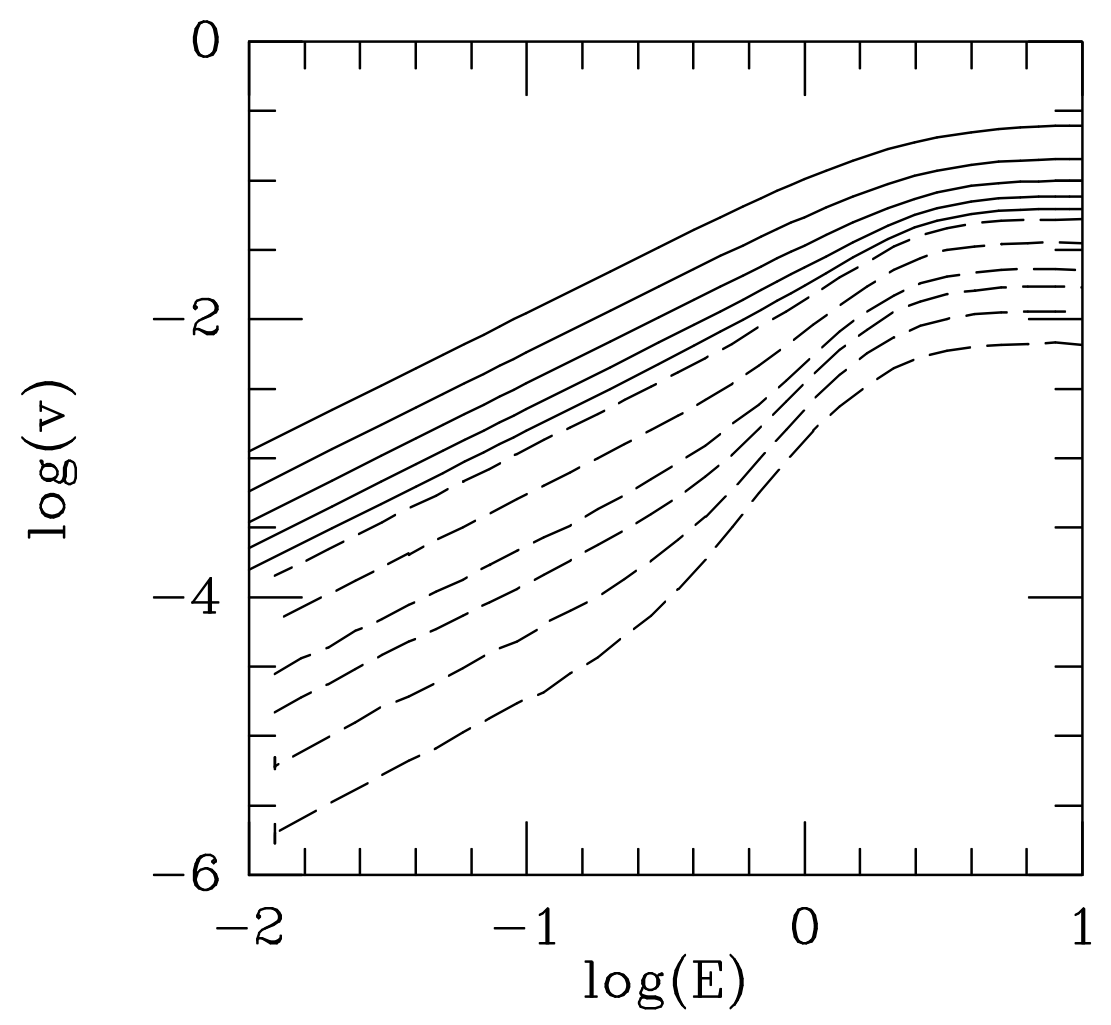

